\title{
Evaluating the roles of green and built-up areas in reducing a surface urban heat island using remote sensing data
}

Rapid urbanization has several negative effects on both the environment and human health. Urbanization has also become an important contributor to global warming. One of these effects is the urban heat island (UHI), which is caused by human activities and defined as the temperature difference between urban and surrounding rural areas. With rapid urbanization in the past few decades, Skopje has experienced remarkable UHI effects. To investigate the roles of built-up and green areas in a surface UHI, this article uses satellite data from Landsat ETM+ to analyse the land surface temperature and high-resolution Planet Scope DOVE data to analyse built-up and green areas. For geostatistical analyses, seventeen randomly selected subareas in Skopje were used.
The results show a significant correlation between the UHI and built-up areas, and strong correlation between green areas and areas not affected by the UHI, indicating that the UHI effect can be significantly weakened with additional green areas. One of the significant findings in the study is the ideal proportion of built-up (40\%) and green areas (60\%), where the UHI effect is weak, or in some cases prevented. For future studies, investigating other factors that may contribute to the UHI phenomenon is suggested.

Keywords: surface urban heat island, remote sensing, satellite imagery, urbanization, land surface temperature 


\section{Introduction}

Rapid population growth in the last few decades has led to drastic urbanization in most parts of the world. Although urbanization is positive for developing countries, if it does not take place correctly it can negatively affect both the environment and human health (Morefield et al., 2018). One of the side effects of urbanization - along with the densification of built-up areas, increase in impervious surfaces, and lack of green spaces - is the appearance of the urban heat island (UHI) phenomenon. This term was first used in 1818 (Bristow et al., 2012). Since the early 1900s, the UHI intensity of hundreds of built-up areas around the world has been assessed (Stewart \& Oke, 2009), and this field remains extensively studied in urban climatology (Souch \& Grimmond, 2006). An UHI is defined as a temperature increase in built-up areas compared to their rural surroundings caused by human activities (Kaplan et al., 2018). If compared with the average temperature of green space and residential areas, the fundamental factor increasing air temperature and surface temperature in urban areas is the difference in solar radiation heating in built-up areas, especially around roads and commercial and industrial areas (Santamouris et al., 2011; Leal Filho et al., 2018). Although many factors affect UHIs, replacing the natural land cover with heat-absorbing surfaces is one of the greatest contributors to this phenomenon (Leal Filho et al., 2018). Based on their formation type, the techniques used to measure them, their impact, and mitigation methods, there are atmospheric and surface UHIs (Munn, 2002).

Whereas atmospheric UHIs are related to air temperature differences between urban and rural (green) areas, surface UHIs (SUHIs) can be defined by the temperature of the entire 3-D envelope of a surface (Fernando et al., 2001). SUHIs are typically present both day and night, but tend to be strongest during the day, when the sun is shining and the city accumulates heat. At night, built-up areas release this heat, and because the original green areas have been replaced by manmade structures the natural cooling effect is lost in the urban areas. Due to changes in the sun's intensity as well as the ground cover, the magnitude of UHI varies with the seasons, and thus SUHIs are typically strongest and highest in summer. The typical identification method for SUHIs is indirect measurements, such as remote sensing methods and techniques.

Over the years, various satellite remote sensing instruments equipped with thermal sensors have been used for mapping and monitoring SUHIs, such as the Moderate Resolution Imaging Spectroradiometer, or MODIS (Miao et al., 2009; Schwarz et al., 2011; Tomlinson et al., 2012), Landsat TM, ETM+ and OLI/TIRS (Zha et al., 2003; Chen et al., 2006;
Van der Hoeven \& Wandl, 2018), and ASTER (Liu \& Zhang, 2011), as well as a combination of these to analyse the Land Surface Temperature (LST) of the study areas. Although low-resolution sensors such as MODIS have been used for large areas, medium-resolution sensors such as Landsat and ASTER are more suitable for observing individual cities or smaller areas. Research on LST and UHIs has shown that surface temperature response is a function of different land cover (Owen et al., 1998), which prompted research on the relationship between LSTs and land cover, and especially vegetation abundance (Gallo \& Owen, 1999; Weng, 2001; Weng et al., 2004; Chen et al., 2006).

In order to evaluate the roles of built-up and green areas in SUHIs, using medium and high spatial remote sensing data, this article addresses several research questions:

- What parts of Skopje are affected by UHIs?

- How are SUHIs, built-up areas, and green areas related?

- What is the ideal proportion of built-up and green areas in a subarea in order to eliminate the SUHI effect?

\section{Methodology}

Skopje is the capital of North Macedonia, located in the Balkan peninsula. The population of the country is estimated at 2.1 million, and the population of Skopje is estimated at more than 800,000 . The city was built in the Skopje Valley, oriented on an east -west axis along the course of the Vardar River, which flows into the Aegean Sea in Greece. Skopje has an elevation of approximately $245 \mathrm{~m}$ and covers an area of approximately $572 \mathrm{~km}^{2}$. The urban expansion of Skopje is limited by mountain ranges to the north and south, and thus the city has developed along the Vardar and the Serava, a small river that flows from the north. Over the last three decades, Skopje has undergone radical changes that have affected urban-planning processes (Stefanovska \& Koželj, 2012). Over the past century, during which Skopje's population has grown more than tenfold, social changes have not only affected planning processes but also impacted the built fabric of the city. As a result, the city has been transformed by a diverse set of dynamics. Skopje is one of many cities that have been affected by the side effects of rapid urbanization. The differences between the temperature in the city and the surrounding areas have been investigated using meteorological measurements and thermal imaging as part of the climate change strategy of the city (City of Skopje, 2017). The results from the meteorological measurements showed differences up to $5^{\circ} \mathrm{C}$ in different parts of the city, and the results from thermal imaging showed a $12^{\circ} \mathrm{C}$ difference between the city centre and the rural surroundings. Kaplan et al. (2018) confirmed the presence of a UHI in Skopje using remote sensing data and also found a 


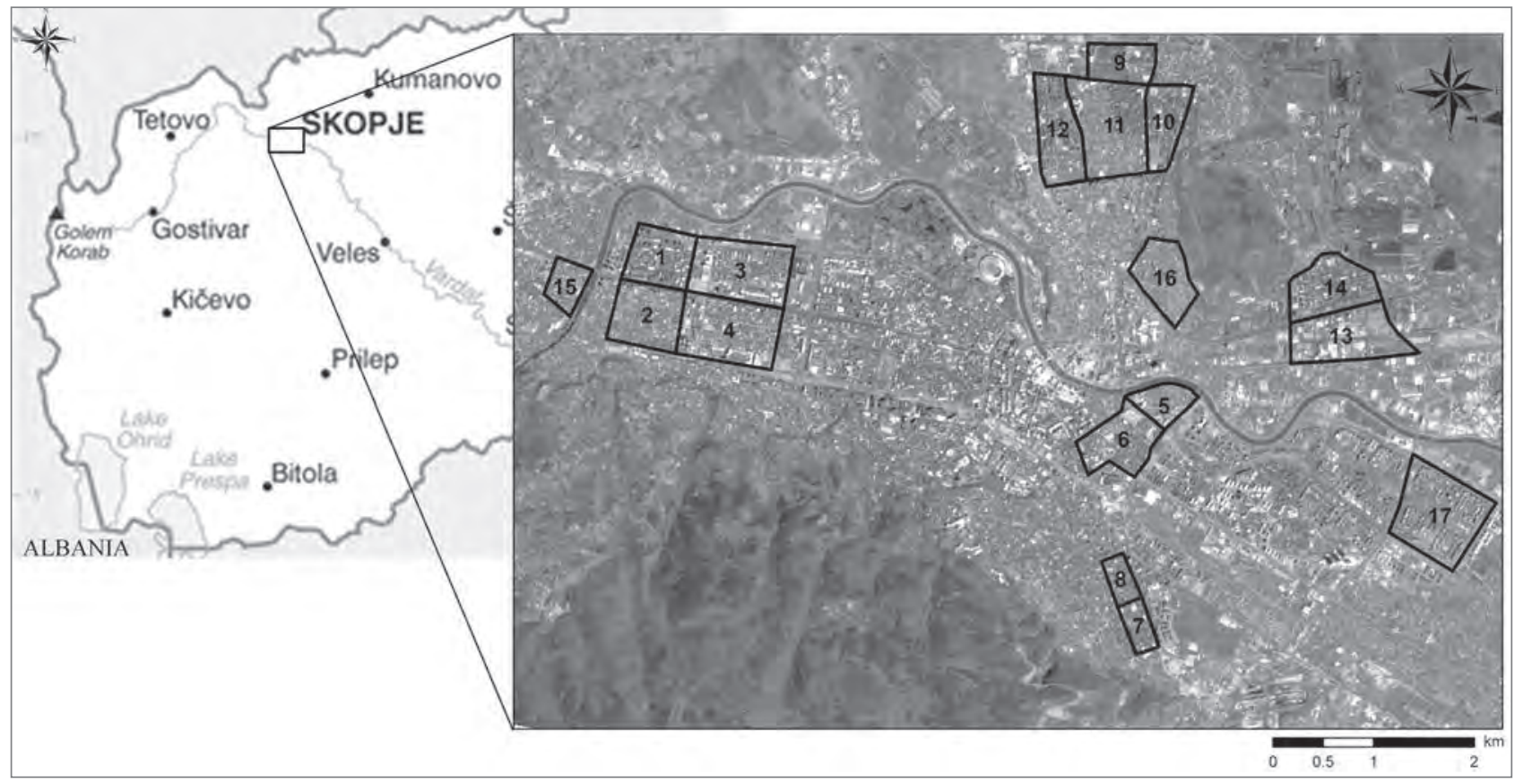

Figure 1: Skopje study area and subareas, DOVE satellite sensor (RGB, 4, 3, 2; author: Gordana Kaplan).

correlation between built-up areas and the UHI. Comparing meteorological data in the period between 2009 and 2019, drastic changes in the temperature can also be noticed. Thus, whereas the minimum temperature in August 2009 was measured at $14^{\circ} \mathrm{C}$, in 2018 and 2019 it had risen to $17^{\circ} \mathrm{C}$ and $19^{\circ} \mathrm{C}$, respectively. Similar is the case with the average and maximum temperatures, where the average temperature rose from $20^{\circ} \mathrm{C}$ to $30^{\circ} \mathrm{C}$, and the maximum from $27^{\circ} \mathrm{C}$ to $32^{\circ} \mathrm{C}$ (WorldWeather, 2019).

In order to analyse the SUHI in Skopje, this study uses a thermal map retrieved from the Landsat ETM+ mapper with medium spatial resolution, and PlanetScope DOVE data were used for extracting the built-up and green areas in the subareas. The Landsat- 7 images acquired since 2003 have a scan line corrector problem and only the middle part (c. $23 \mathrm{~km})$ of each scene has no data loss. Because this part fully covers the study area used in this article, no additional corrections were made. The PlanetScope DOVE data were downloaded from the Planet webpage, which operates with more than 175 PS satellites that collect multispectral imagery in four bands with a collection capacity of 300 million $\mathrm{km}^{2}$ per day. The sensor used in this study collects four multispectral bands (NIR, RGB) with $3 \mathrm{~m}$ spatial resolution. In order to extract the urban and green areas from the high-resolution data in the subareas, a normalized difference vegetation index (NDVI, Equation 1) was used.

$$
N D V I=\frac{N I R-R e d}{N I R+R e d}
$$

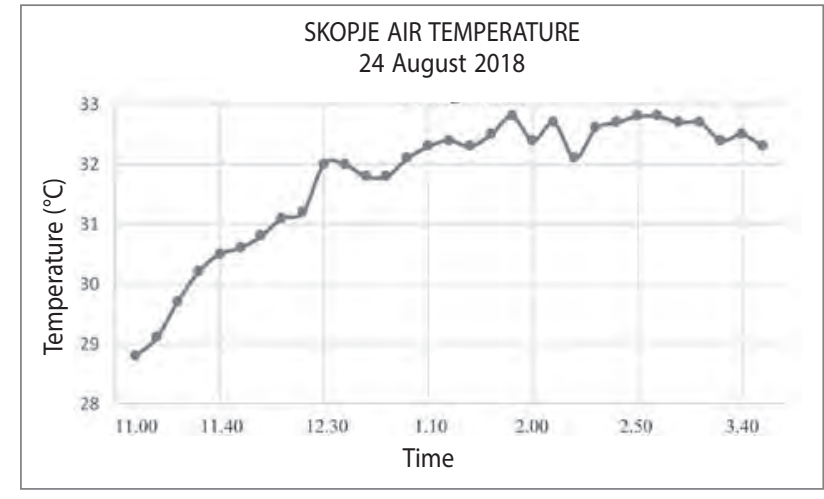

Figure 2: Skopje air temperature on 24 August 2018 (author: Gordana Kaplan).

Because Skopje has a continental-sub-Mediterranean climate, meaning the temperatures are high in the summer, this article uses satellite images acquired at the end of August 2017.

Both Landsat ETM+ and PlanetScope DOVE data were acquired in the summer, on 24 August 2018, when it was assumed that the SUHI effect is the strongest. In addition, to compare the LST and air temperature, air temperature data acquired in several locations in Skopje on the same date were used (Figure 2). As seen from Figure 2, the maximum temperature on the same day that the area was overpassed by the satellites was approximately $33^{\circ} \mathrm{C}$.

As already stated, SUHIs can be best observed through thermal satellite sensors with LST data. The LST can be calculated using the Landsat thermal bands. Several studies can be found 


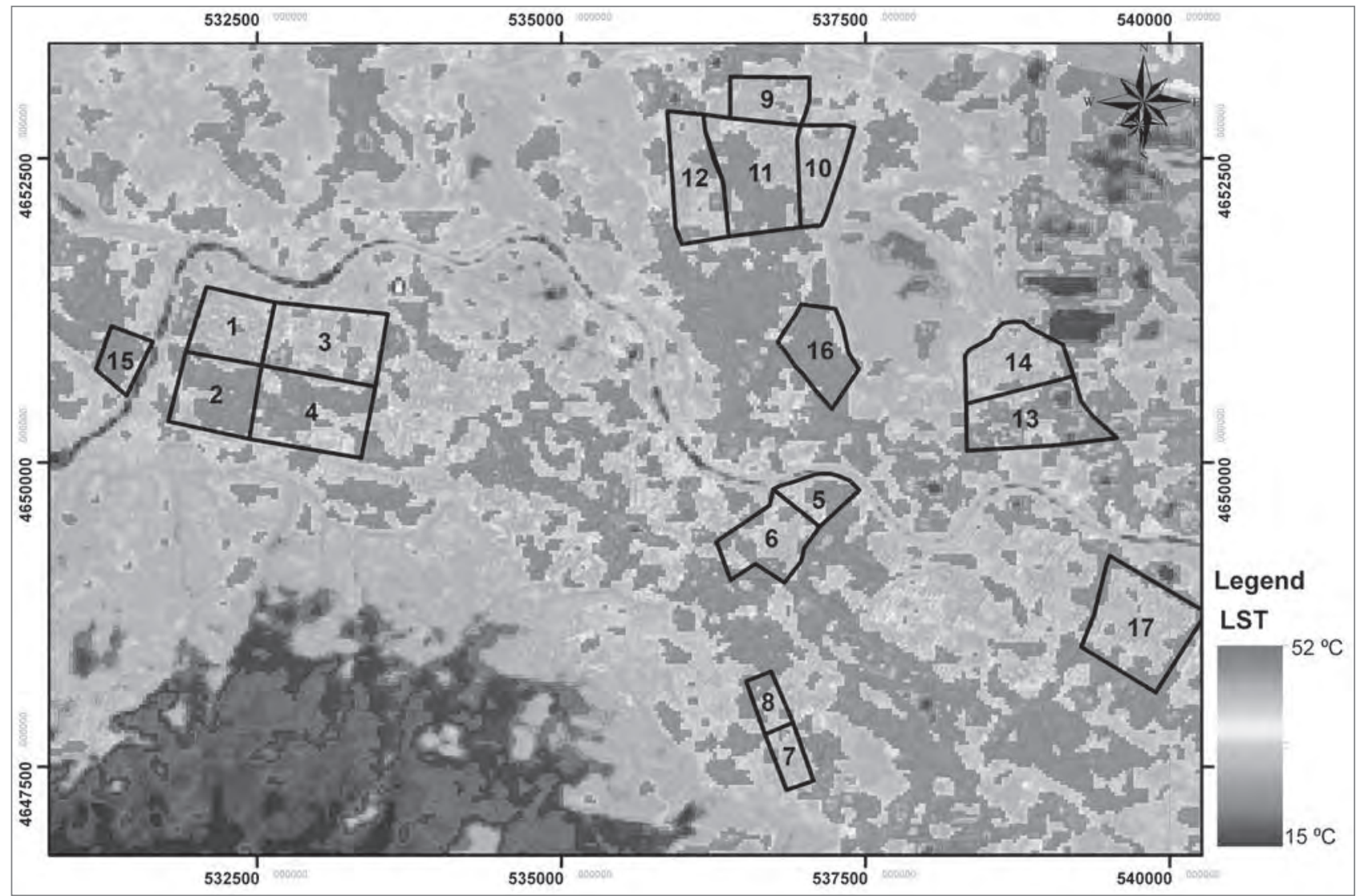

Figure 3: LST, SUHI map (Landsat ETM+) of Skopje (author: Gordana Kaplan).

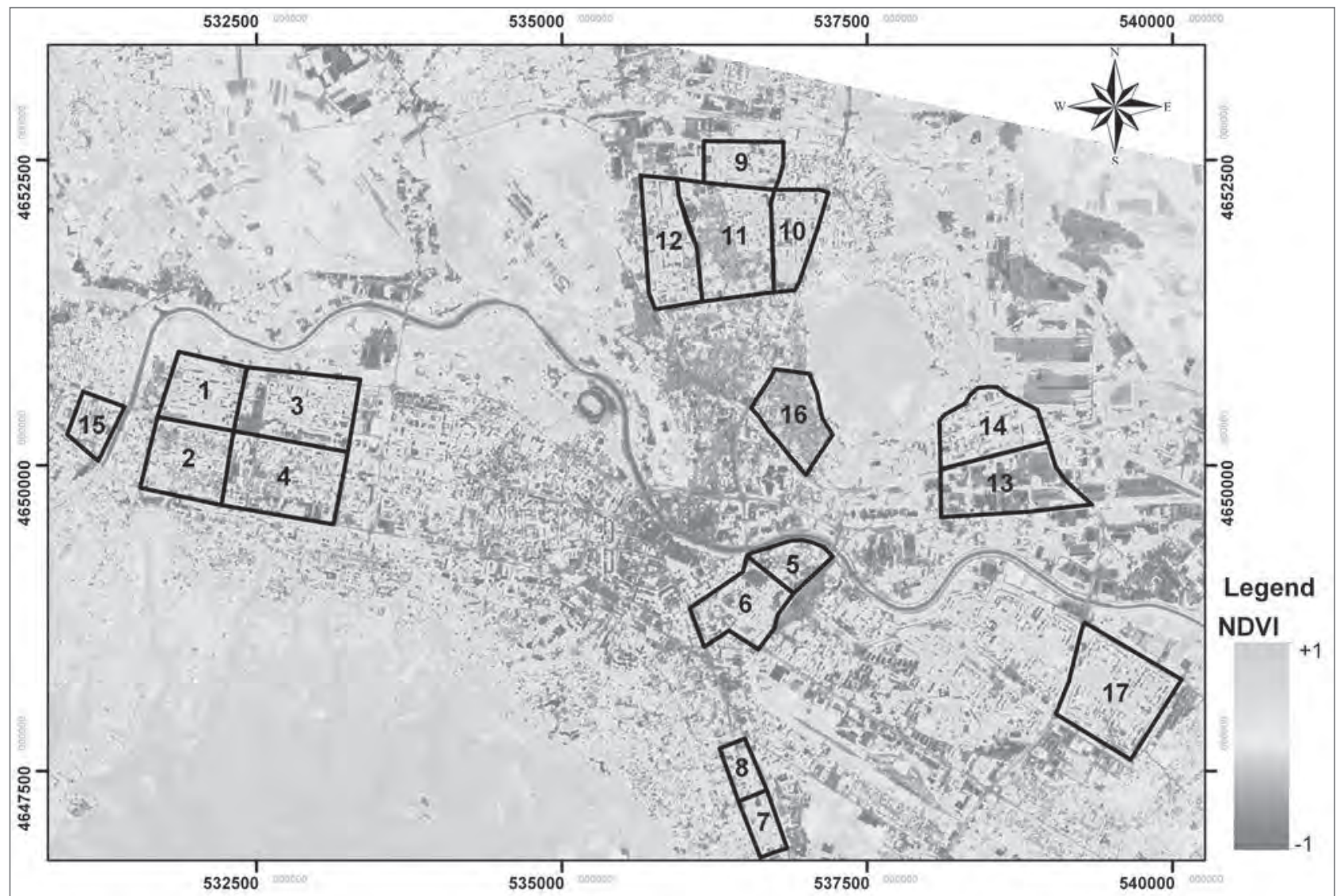

Figure 4: NDVI map of Skopje (author: Gordana Kaplan). 
Table 1: Details of the satellite data used.

\begin{tabular}{lll}
\hline Detail & Satellite & \\
\hline & Landsat ETM+ & PlanetScope DOVE \\
\hline Resolution & $30 \mathrm{~m}$ & $3 \mathrm{~m}$ \\
\hline Bands & 8 & 4 \\
\hline Acquisition date & 24 Aug. 2018 & 24 Aug. 2018 \\
\hline Data used & LST & NDVI \\
\hline
\end{tabular}

on this topic in the literature (Ekercin et al. 2016). This article uses an algorithm developed by Avdan and Jovanovska (2016) that was properly adjusted for retrieving the LST map from Landsat ETM+. The adjustment was made by updating the coefficients of the thermal band that can be found in the metadata of the satellite image.

$$
\begin{aligned}
& K 1_{\text {kanal } 6}=666,09 \mathrm{Wm}^{-2} s r^{-1} \mu \mathrm{m}^{-1} \\
& K 2_{\text {kanal } 6}=1.282 .71 \mathrm{~K}
\end{aligned}
$$

The comparison between the data used was made based on area results. Thus, after the LST map was retrieved, an SUHI map was extracted. Because an SUHI is defined as a temperature difference between the rural and urban areas, an SUHI can be calculated with simple equations. Ma et al. (2010) proposed the following equation for calculating an SUHI, which has been successfully used in many studies (Kaplan et al., 2018):

$$
S U H I=\mu+\frac{\sigma}{2}
$$

where $\mu$ is the mean LST value of the study area and $\sigma$ is the standard deviation of the LST. Areas that have higher temperature values than the SUHI are considered areas highly affected by the UHI phenomenon.

Afterward, statistical analyses of the SUHI area and the builtup and green areas were made based on the reclassified images according to the LST and SUHI analyses. Thus, both green and built-up areas were extracted from the PlanetScope DOVE satellite image (Planet Team, 2017), and the LST, SUHI, and areas not affected by UHIs were extracted as areas in $\mathrm{m}^{2}$ and then statistically and visually compared. Details of the data are given in Table 1.

\section{Results and discussion}

Similar to the results of the previous study, the results of this study indicate the presence of an SUHI in several parts of Skopje. The SUHI analyses showed that areas with LSTs higher than $34^{\circ} \mathrm{C}$ are areas affected by the SUHI (Figure 3). The results show that the SUHI effect is usually the strongest in the dense built-up areas, which can be confirmed by the results

\begin{tabular}{|c|c|c|c|}
\hline ID no. & Urban & Green & SUHI \\
\hline 1 & 35.64 & 64.36 & 7.82 \\
\hline 2 & 65.96 & 34.04 & 71.14 \\
\hline 3 & 54.40 & 45.60 & 24.87 \\
\hline 4 & 69.89 & 30.11 & 57.49 \\
\hline 5 & 77.96 & 22.04 & 45.96 \\
\hline 6 & 63.54 & 36.46 & 39.21 \\
\hline 7 & 28.61 & 71.39 & 8.58 \\
\hline 8 & 56.38 & 43.62 & 44.65 \\
\hline 9 & 40.35 & 59.65 & 9.81 \\
\hline 10 & 35.66 & 64.34 & 8.12 \\
\hline 11 & 63.82 & 36.18 & 51.26 \\
\hline 12 & 46.39 & 53.61 & 21.53 \\
\hline 13 & 75.16 & 24.84 & 73.32 \\
\hline 14 & 42.71 & 57.29 & 12.41 \\
\hline 15 & 66.47 & 33.53 & 76.14 \\
\hline 16 & 89.10 & 10.90 & 85.89 \\
\hline 17 & 39.56 & 60.44 & 31.79 \\
\hline
\end{tabular}
of the NDVI analyses shown in Figure 4.
Table 2: Results of geostatistical analyses (\%).

For every subarea of Skopje, four parameters were extracted: built-up and green areas, and areas affected and not affected by the SUHI phenomenon (Table 2). The results showed a significantly high correlation between built-up areas and the SUHI $(r=0.92)$, and a high correlation between green canopy areas and areas not affected by the SUHI $(r=0.95)$. In addition, the coefficients of determination for the same comparisons are also significantly high, indicating that more than $84 \%$ of the SUHI can be explained by the linear relation with the built-up areas (Figure 5), and 90\% of the areas not affected by UHIs can be explained by the linear relation with the green canopy in the study areas (Figure 5).

As can be seen from the results, UHIs are strongly connected with built-up areas in a specific neighbourhood. Thus, an ideal proportion should be found whereby the built-up area does not cause the UHI effect, or at least weakens its effects. For this purpose, the results were also converted into percentage values and statistical analyses were made. In most of the cases, the study areas that were covered by more than $40 \%$ built-up area showed strong SUHI effects (Figure 6). The visual comparison, however, showed that the $40 \%$ needs to be equally distributed across the area. 

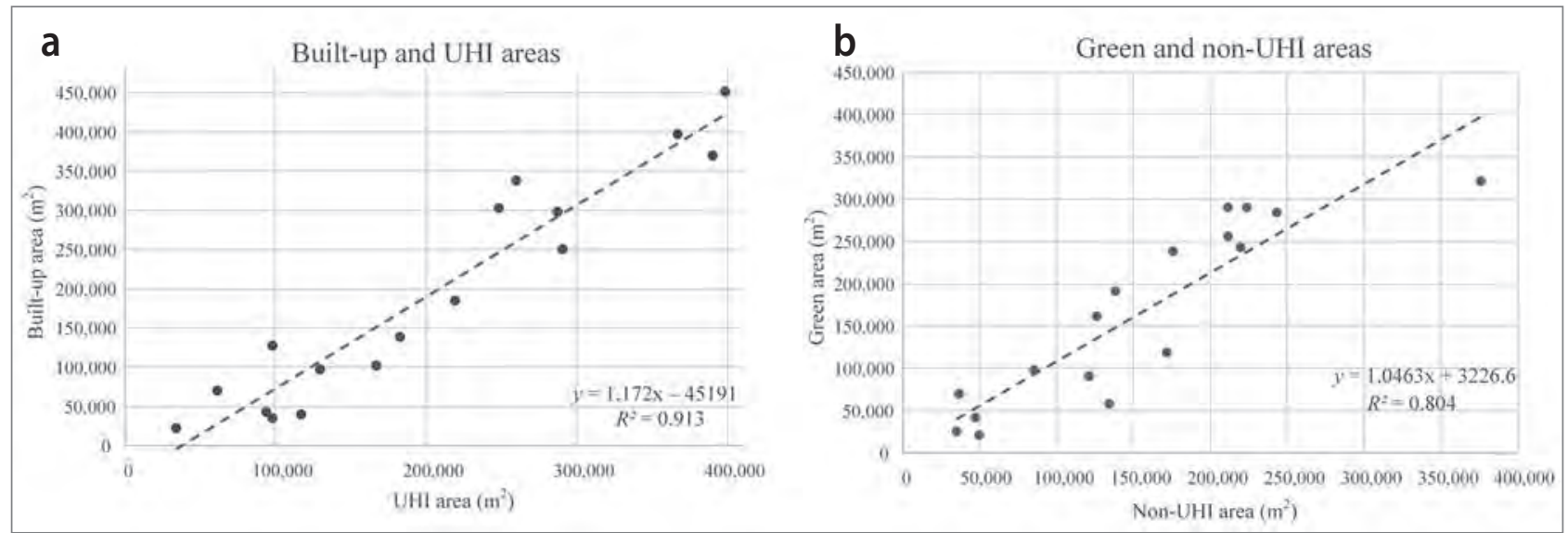

Figure 5: a) built-up area and UHI correlation; b) green canopy and non-UHI correlation (author: Gordana Kaplan).

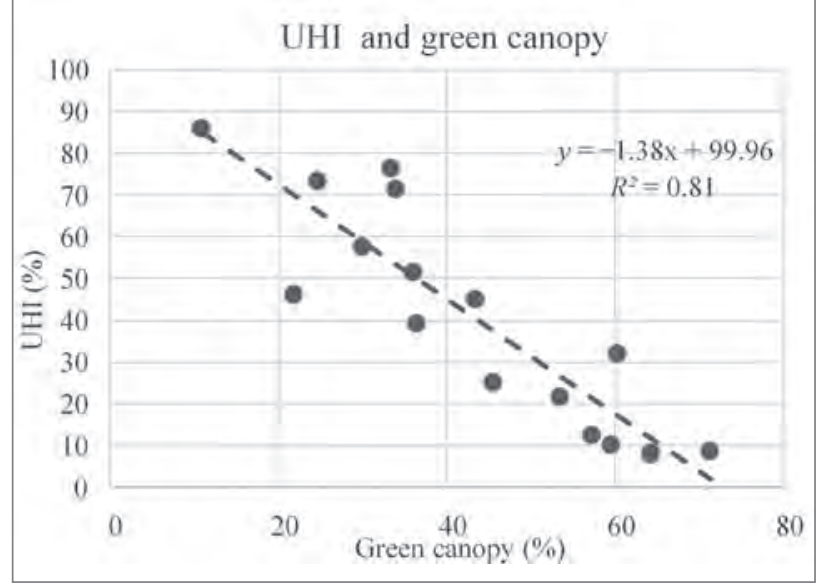

Figure 6: UHI and green canopy correlation (author: Gordana Kaplan).

As seen from Figure 6, the UHIs and green canopy areas are inversely correlated, which means that UHIs can be weakened with green areas. However, this is the case for $81 \%$ of the areas studied, meaning there are other factors that affect areas with the UHI phenomenon. As can be seen from the visual comparisons, one of these factors is the density of the built-up area. For example, whereas subareas numbers 6 and 11 have approximately same percentage of built-up area $(63.5 \%$ and $63.8 \%$ respectively), subarea 11 has approximately $11 \%$ more area affected by an UHI due to built-up area density. The best results were seen in subareas $1,7,9$, and 10 , where the UHI areas were lower than $10 \%$ and the built-up areas did not exceed $40 \%$.

The results of this study confirm the current situation in Skopje, where the temperature differences between the city and the surrounding areas are up to $12^{\circ} \mathrm{C}$. The UHI phenomenon can also be noticed from the meteorological data in the period between 2009 and 2019, where there is a difference up to $10^{\circ} \mathrm{C}$ on average. These changes have been noticed by the authorities, and since 2016 detailed investigations on this topic have been ongoing. At the end of 2018, the authorities published an action plan for mitigating UHIs in Skopje. The action plan was created based on the guidelines of the United States Environmental Protection Agency, which is responsible for UHI mitigation in the United States. The main strategies for reducing UHIs are increasing green areas, installing green roofs, cool reflective roofs, and cool pavements, and utilizing smart growth practices. The action plan also aims to plant one million trees. The results of this study may be useful for determining in advance areas for planting trees and other green areas.

\section{Conclusion}

This article investigated the SUHI phenomenon in Skopje using Landsat ETM+ imagery and evaluated the urban and green canopy roles in SUHI intensity using high-resolution imagery from the Planet Scope DOVE satellite. Because the presence of a UHI in Skopje was confirmed in another study (Kaplan et al., 2018), this article analysed seventeen randomly selected subareas, seeking to answer several research questions. The areas differed in size, location, and UHI effect. Thus, in order to investigate the relation between the urban areas, green areas, and areas affected by UHIs, several geostatistical analyses were carried out. The results of this study indicate that several parts of Skopje have been affected by the UHI phenomenon.

As expected, the results showed a high correlation between the SUHI and the built-up area $\left(R^{2}=84\right)$, indicating that the SUHI is caused by human activities in the study area. Proportionally, there is a strong correlation between the green and non-UHI areas $\left(R^{2}=90\right)$, indicating that a green canopy can significantly weaken the UHI/SUHI effect. A comparison with the meteorological data collected on the same day the satellite carrying a thermal sensor overpassed the area showed that the SUHI is present in areas where the land surface temperature is higher than the measured air temperature. 
The results of this study indicate that $81 \%$ of the time UHIs in Skopje are caused by human activities or built-up areas. Another factor affecting UHIs is the density of the built-up and green areas. This study showed that ideal urban planning with which UHIs can be avoided or strongly weakened comprises $40 \%$ built-up space and $60 \%$ green canopy. In order to avoid a UHI effect, built-up areas should be separated from each other and surrounded by a green canopy. This study can be used by both city planners and environmental engineers in decision-making for mitigating UHI effects, which can be harmful to both the environment and human health. For future studies, it is recommended that further investigations be carried out of other factors that may be affecting built-up areas with a UHI. It is also recommended that the ratio be determined between green and built-up areas for mitigating UHIs in other study areas.

Gordana Kaplan

Eskisehir Technical University, Earth and Space Sciences Institute,

Eskisehir, Turkey

E-mail: kaplangorde@gmail.com

\section{Acknowledgments}

The author thanks Planet Labs, Inc. for providing high-resolution Planet Scope imagery of Skopje.

\section{References}

Avdan, U. \& Jovanovska, G. (2016) Algorithm for automated mapping of land surface temperature using LANDSAT 8 satellite data. Journal of Sensors, 2016, Article ID 1480307. DOI: 10.1155/2016/1480307

Bristow, R. S., Blackie, R. \& Brown, N. (2012) Parks and the urban heat island: A longitudinal study in Westfield, Massachusetts. In: Fisher, C. L. \& Watts, C. E., Jr. (eds.) Proceedings of the 2010 Northeastern Recreation Research Symposium. Gen. Tech. Rep. NRS-P-94, pp. 224-230. Newtown Square, PA, U.S. Department of Agriculture, Forest Service, Northern Research Station. DOI: 10.1007/s10980-010-9515-9

Chen, X.-L., Zhao, H.-M., Li, P.-X. \& Yin, Z.-Y. (2006) Remote sensing image-based analysis of the relationship between urban heat island and land use/cover changes. Remote Sensing of Environment, 104(2), pp. 133-146. DOI: 10.1016/j.rse.2005.11.016

City of Skopje (2017) Resilient Skopje, Climate change strategy report. Skopje.

Ekercin, S., Orhan, O. \& Dadaser-Celik, F. (2016) Investigating land surface temperature changes using Landsat- 5 data and real-time infrared thermometer measurements at Konya closed basin in Turkey. International Journal of Engineering and Geosciences, 4(1), pp. 16-27. DOI: $10.26833 /$ ijeg.417151

Fernando, H., Lee, S., Anderson, J., Princevac, M., Pardyjak, E. \& Grossman-Clarke, S. (2001) Urban fluid mechanics: Air circulation and contaminant dispersion in cities. Environmental Fluid Mechanics, 1(1), pp. 107-164. DOl: 10.1023/a:1011504001479

Gallo, K. P. \& Owen, T. W. (1999) Satellite-based adjustments for the urban heat island temperature bias. Journal of Applied Meteorology, 38(6), pp. 806-813. DOI: 10.1175/1520-0450(1999)038<0806:sbaftu>2.0.co;2
Kaplan, G., Avdan, U. \& Avdan, Z. Y. (2018) Urban heat island analysis using the Landsat 8 satellite data: A case study in Skopje, Macedonia. Skopje, Multidisciplinary Digital Publishing Institute Proceedings. DOI: 10.3390/ecrs-2-05171

Leal Filho, W., Icaza, L. E., Neht, A., Klavins, M. \& Morgan, E. A. (2018) Coping with the impacts of urban heat islands. A literature based study on understanding urban heat vulnerability and the need for resilience in cities in a global climate change context. Journal of Cleaner Production, 171, pp. 1140-1149. DOI: 10.1016/j.jclepro.2017.10.086

Liu, L. \& Zhang, Y. (2011) Urban heat island analysis using the Landsat TM data and ASTER data: A case study in Hong Kong. Remote Sensing, 3(7), pp. 1535-1552. DOI: 10.3390/rs3071535

Ma, Y., Kuang, Y. \& Huang, N. (2010) Coupling urbanization analyses for studying urban thermal environment and its interplay with biophysical parameters based on TM/ETM+ imagery. International Journal of Applied Earth Observation and Geoinformation, 12(2), pp. 110-118. DOI: 10.1016/j.jag.2009.12.002

Miao, S., Chen, F., LeMone, M. A., Tewari, M., Li, Q. \& Wang, Y. (2009) An observational and modeling study of characteristics of urban heat island and boundary layer structures in Beijing. Journal of Applied Meteorology and Climatology, 48(3), pp. 484-501. DOI: 10.1175/2008jamc1909.1

Morefield, P., Fann, N., Grambsch, A., Raich, W. \& Weaver, C. (2018) Heat-related health impacts under scenarios of climate and population change. International Journal of Environmental Research and Public Health, 15(11), p. 2438. DOl: 10.3390/ijerph15112438

Munn, T. (2002) Encyclopedia of global environmental change. Chichester, UK, Wiley.

Owen, T., Carlson, T. \& Gillies, R. (1998) An assessment of satellite remotely-sensed land cover parameters in quantitatively describing the climatic effect of urbanization. International Journal of Remote Sensing, 19(9), pp. 1663-1681. DOI: 10.1080/014311698215171

Planet Team (2017) Planet application program interface: In space for life on Earth. San Francisco, CA. Available at: https://api.planet.com (accessed 21 Sept. 2019).

Santamouris, M., Synnefa, A. \& Karlessi, T. (2011) Using advanced cool materials in the urban built environment to mitigate heat islands and improve thermal comfort conditions. Solar Energy, 85(12), pp. 30853102. DOI: 10.1016/j.solener.2010.12.023

Schwarz, N., Lautenbach, S. \& Seppelt, R. (2011) Exploring indicators for quantifying surface urban heat islands of European cities with MODIS land surface temperatures. Remote Sensing of Environment, 115(12), pp. 3175-3186. DOI: 10.1016/j.rse.2011.07.003

Souch, C. \& Grimmond, S. (2006) Applied climatology: Urban climate. Progress in Physical Geography, 30(2), pp. 270-279. DOI: 10.1191/0309133306pp484pr

Stefanovska, J. \& Koželj, J. (2012) Urban planning and transitional development issues: The case of Skopje, Macedonia. Urbani izziv, 23(1), pp. 91-100. DOI: 10.5379/urbani-izziv-en-2012-23-01-002

Stewart, I. \& Oke, T. (2009) Newly developed "thermal climate zones" for defining and measuring urban heat island magnitude in the canopy layer. Paper presented at the Eighth Symposium on Urban Environment, 11-15 January, Phoenix, AZ. Typescript.

Tomlinson, C., Chapman, L., Thornes, J. and Baker, C. (2012) Derivation of Birmingham's summer surface urban heat island from MODIS satellite images. International Journal of Climatology, 32(2), pp. 214-224. DOI: $10.1002 /$ joc.2261 
Van der Hoeven, F. \& Wandl, A. (2018) Hotterdam: Mapping the social, morphological, and land-use dimensions of the Rotterdam urban heat island. Urbani izziv, 29(1), pp. 58-72.

DOI: 10.5379/urbani-izziv-en-2018-29-01-001

Weng, Q. (2001) A remote sensing? GIS evaluation of urban expansion and its impact on surface temperature in the Zhujiang Delta, China. International Journal of Remote Sensing, 22(10), pp. 1999-2014. DOI: 10.1080/01431160152043676

Weng, Q., Lu, D. \& Schubring, J. (2004) Estimation of land surface temperature-vegetation abundance relationship for urban heat island studies. Remote Sensing of Environment, 89(4), pp. 467-483. DOI: 10.1016/j.rse.2003.11.005

WorldWeather (2019) Skopje monthly climate averages. Available at: https://www.worldweatheronline.com/skopje-weather-averages/karpos/ mk.aspx (accessed 21 Sept. 2019).

Zha, Y., Gao, J. \& Ni, S. (2003) Use of normalized difference builtup index in automatically mapping urban areas from TM imagery. International Journal of Remote Sensing, 24(3), pp. 583-594. DOI: 10.1080/01431160304987 